\title{
Reducing Interpolation Artifacts for Mutual Information Based Image Registration
}

\author{
Hossein Soleimani, Mohammadali Khosravifard \\ Department of Electrical and Computer Engineering, Isfahan University of Technology, 84156-83111 Isfahan, Iran
}

\section{A B S T R A C T}

Medical image registration methods which use mutual information as similarity measure have been improved in recent decades. Mutual information is a basic concept of information theory which indicates the dependency of two random variables (or two images). In order to evaluate the mutual information of two images, their joint probability distribution is required. Several interpolation methods, such as Partial Volume (PV) and bilinear, are used to estimate joint probability distribution. Both of these two methods yield some artifacts on mutual information function. Hanning window- Partial Volume and Generalized Partial Volume methods are introduced to remove such artifacts. In this paper, we show that the acceptable performance of these methods is not due to their kernel function. It's because of the number of pixels which incorporate in interpolation. Since using more pixels requires more complex and time consuming interpolation process, we propose a new interpolation method which uses only four pixels (the same as PV and bilinear interpolations) and removes most of the artifacts. Experimental results of the registration of Computed Tomography images show superiority of the proposed scheme.

Key words: Artifact, image registration, interpolation, mutual information

\section{INTRODUCTION}

Image registration is the alignment of two or more different images of a view or an object such that their similar pixels coincide to each other. These images might be acquired by several sensors or by a single sensor in different viewpoints, moments or other different conditions. The goal of image registration is to find a transform function by which the floating image is aligned to the reference image. Every image registration algorithm requires three basic ingredients: 1 ) a spatial transformation model which determines the set of possible solutions, 2) an objective similarity measure which estimates the quality of each potential solution, and 3) an optimization algorithm which looks for the best solution. ${ }^{[1,2]}$

There are numerous medical imaging modalities that show the anatomy or morphology. Especially, computed tomography (CT) and magnetic resonance (MR) imaging of the head for diagnosis and surgical issues, provide significant information for surgeons. To obtain more complete information about the patient, monitoring tumor growth and comparison of the patient's data with anatomical information, registration between these modalities is necessary. ${ }^{[2,3]}$

Multimodality registration is of great importance in many medical applications. Over the years, many different methods are advised for this task. A complete survey of these methods and classification of approaches can be found in..$^{[4,5]}$

Image registration methods are classified to 1) intensity and 2) feature based methods. Feature based methods use some features (edge, surface, and line etc), which requires segmentation and feature selection. Intensity based methods, which are more popular in medical image registration, ${ }^{[6]}$ employ the intensity of pixels and no feature is needed.

Mutual information is an information-theoretic concept which has been widely used as the similarity measure in intensity based methods. It shows high accuracy and robustness with intensity variation and noise. ${ }^{|7,8|}$ Mutual information is also employed in other image processing applications such as template matching ${ }^{[9]}$ and object tracking. ${ }^{[10]}$

The most important and critical step in calculating the mutual information of two random variables (or two images) is to find their joint probability distribution. Since the acquired images are actually samples of continuous two-dimensional signals, calculation of their joint probability distribution cannot be perfect. Thus, an interpolation method should be employed for estimating 
the joint probability distribution which influences the quality of image registration. Therefore, selection of a reliable interpolation method for estimating the joint probability distribution is of great importance.

The joint probability distribution can be derived by using Partial Volume (PV) or bilinear interpolation methods. ${ }^{[11]}$ When the underlying images are acquired by a single sensor, and hence the size of their pixels is equal, mutual information (as a function of translation) shows some local optima at integer values. ${ }^{[12]}$ These artifacts may cause the optimization algorithm to converge to wrong solutions. This will degrade the accuracy of the registration.

In order to remove such artifacts, Hanning windowPartial Volume (HPV) and generalized partial volume (GPV) interpolation methods are proposed. ${ }^{[5,13]}$ With these methods, interpolation procedure is performed by using Hanning windowed sinc and Spline as kernel functions instead of boxcar function used in PV interpolation. In, ${ }^{[5]}$ it is shown that the performance of these methods is better than that of PV. But this comparison is questionable, since in HPV and GPV, 16 pixels are involved in the interpolation, while for PV, only 4 pixels are involved. As a result, one cannot conclude that the superiority of HPV and GPV is due to their appropriate kernel functions. In contrast, in this paper, we show that the importance of the number of involved pixels in the performance of registration is more than the selected kernel function. Also, we propose a new interpolation method which uses only four pixels with better performance with respect to PV and bilinear interpolations. Since the resulted mutual information function is smoother, the registration is more accurate.

The paper is organized as follows. In Section 2, the problem of generated artifacts in mutual information function is stated. In Section 3, different interpolation methods are explained. The main idea of the paper is also discussed in Section 3. Finally, in Section 4, performance of PV, bilinear, and proposed methods are compared.

\section{ARTIFACTS IN MUTUAL INFORMATION FUNCTION}

Mutual information is one of the basic concepts of information theory which indicates the dependency of two random variables. Initially, it was used in medical image registration by Viola and Wells in 1995..$^{[14]}$

It is well-known that two random variables, $A$ and $B$, with marginal probability distributions $p_{A}(a)$ and $p_{B}(b)$ and joint probability distribution $p_{A, B}(a, b)$ are independent iff $p_{A, B}(a, b)=p_{A}(a) \cdot p_{B}(b)$. Mutual information $I(A, B)$ measures the dependency of $A$ and $B$ through the Kullback-Leibler distance of the distributions $p_{A, B}(a, b)$ and $p_{A}(a) \cdot p_{B}(b),{ }^{[15]}$ i.e.
$I(A, B)=\sum_{a, b} p_{A, B}(a, b) \cdot \log \left(\frac{p_{A, B}(a, b)}{p_{A}(a) \cdot p_{B}(b)}\right)$

Probability distributions of images are easily derived by normalizing the correspondent histograms. Mutual information takes its maximum value when the underlying random variables (or images) are absolutely dependent (one of them is a function of the other one). In such case, the joint probability matrix (or joint histogram matrix) is diagonal. Reversely, if the images are independent, then the mutual information takes its minimum value, i.e. zero.

In order to align two images, a transformation function $T$ should be applied to the floating one. Transforming a grid point of floating image usually gives a point in the reference image which does not coincide with a grid point. Thus, an interpolation method is required to estimate the joint histogram of the images.

The prevalent interpolation method in image processing is bilinear interpolation, which uses four nearest pixels of the transformed coordinate in the reference image. Joint histogram is easily obtained by a simple counting procedure. ${ }^{[1]}$ With this method, the resulted mutual information function is not smooth and the optimization algorithm may converge to a wrong solution. The situation is more critical when the images are noisy.

In order to overcome this problem, PV interpolation method was proposed. But it has been shown in ${ }^{[12 \mid}$ that if the underlying images have the same pixel size, PV and bilinear interpolation yield some local optima (artifacts) on the surface of mutual information function. A typical pattern of mutual information function in terms of displacement (translation) in horizontal and vertical directions is shown in Figure 1. It can be seen that the surface has some local minimums (res. maximums) for bilinear (res. PV) interpolation method.

\section{INTERPOLATION METHODS AND PROPOSED ALGORITHM}

In this section, first we describe some interpolation

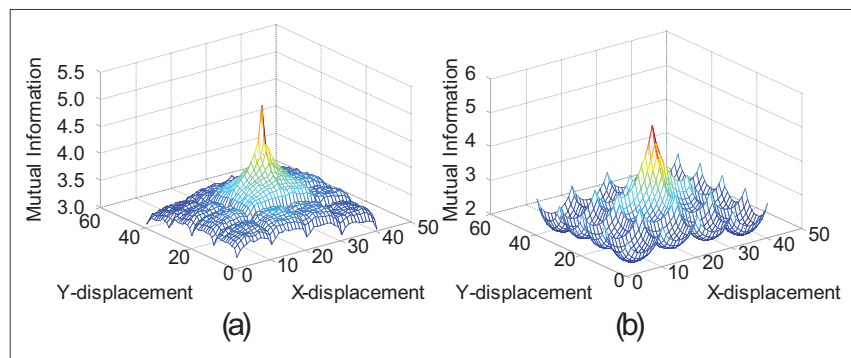

Figure I: Typical patterns of local extremes of mutual information resulting from two interpolation methods. (a) PV; (b) Bilinear 
methods such as bilinear, PV, and its modifications. We investigate the reason behind the acceptable performance of GPV and HPV methods. Also, an interpolation scheme is proposed.

\section{Bilinear Interpolation}

As mentioned in the previous section, transforming coordinate of a pixel of floating image $F$ gives a new coordinate in the reference image $R$, which may not be a grid point. Hence, the intensity of such point must be somehow interpolated. In bilinear interpolation, the intensity is estimated by computing a weighted average of the intensity of four nearest pixels in the reference image.

If the point $p$ with coordinate $s$ in the floating image is transformed (by transformation $T$ ) to point $q$ with coordinate $T_{S}$ in the reference image [Figure 2], the intensity of the reference image in coordinate $T_{S}$ is given by

$$
\begin{array}{rlr}
R\left(T_{s}\right) & =\sum_{i=1}^{4} w_{i} R\left(n_{i}\right) & \\
w_{1}=\left(1-d_{1}\right)\left(1-d_{2}\right) & w_{2}=d_{1}\left(1-d_{2}\right) \\
w_{3}=\left(1-d_{1}\right) d_{2} & w_{4}=d_{1} d_{2}
\end{array}
$$

Where, $n_{i}, i=1,2,3,4$ denote the coordinate of four nearest grid points to point $q$ in the reference image. Note that we have $\sum_{i=1}^{4} w_{i}=1$.

For each point $p$ of the floating image the command $h\left(R\left(T_{s}\right), F(s)\right)+=1$ is used to estimate the joint histogram of floating and reference images. Since bilinear interpolation does not show acceptable robustness to noise, ${ }^{[6]}$ other methods such as PV are more popular.

\section{PV, GPV, HPV and the Proposed Interpolation Methods}

PV method is not actually an interpolation scheme for estimating the intensity in a non-grid point. It is only designed to estimate the joint histogram of two images. Instead of interpolating the intensity values in $R$, the contribution of the intensity of the point $p$ to the joint histogram is distributed over the intensity of all four nearest neighbors of $q$. Updating the joint histogram of $F$ and $R$ is performed by $h\left(R\left(n_{i}\right), F(s)\right)+=w_{i}$ where

$w_{i}=f\left(d_{1 i}\right) f\left(d_{2 i}\right) \quad, \quad i=1,2,3,4$

and $n_{i}, i=1,2,3$, and 4 denotes the four nearest grid points to point $q$ in the reference image. Also, $d_{1 i}$ and $d_{2 i}$ stands for the horizontal and vertical distance of $q$ from $n_{i}$.

With the PV method, the kernel function is defined by:

$f_{P V}(x)=1-x \quad, \quad 0<x<1$ which is known as Boxcar function. In ${ }^{|9|}$ Chen and Varshney proposed GPV interpolation method. They replaced Boxcar function with a third order Spline, i.e.,

$$
f_{G P V}(x)= \begin{cases}\frac{x^{3}}{2}-x^{2}+\frac{2}{3} & 0 \leq x<1 \\ \frac{(2-x)^{3}}{6} & 1 \leq x<2 \\ 0 & \text { otherwise }\end{cases}
$$

In this method, sixteen pixels are used to calculate $w_{i}$ 's and update the joint histogram. As a result, for each pixel of floating image, sixteen bins are updated in the joint histogram. Note that the support of this function is the interval $(0,2)$.

In, ${ }^{[8]} \mathrm{HPV}$ interpolation method was proposed by replacing the boxcar function with Hanning windowed sinc function

$f_{H P V}(x)=\left\{\begin{array}{lc}\frac{1+\cos (2 \pi x / 4)}{4} & 0 \leq x<2 \\ 0 & \text { otherwise }\end{array}\right.$

Similar to GPV method, sixteen pixels are used to estimate the joint histogram. The kernel functions $f_{P V}, f_{G P V}, f_{H P V}$ are shown in Figure 3.

It can be seen from that their behavior is almost the same. Thus, we can expect to achieve the same performance with

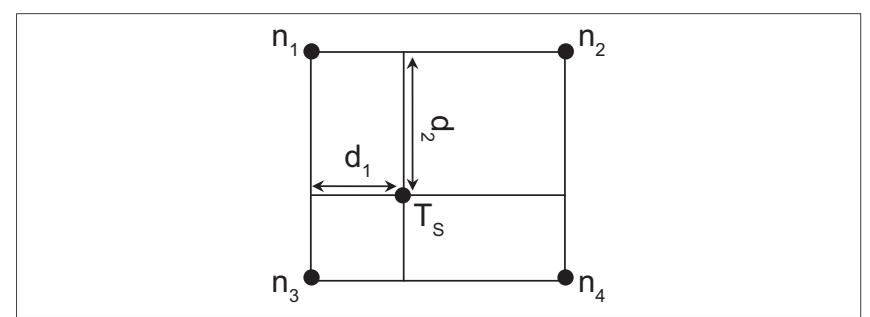

Figure 2: Scheme of transformed pixel from floating image with coordinate $T_{s}$ in reference image and four nearest pixels

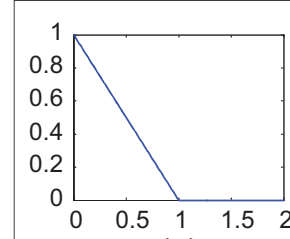

(a)

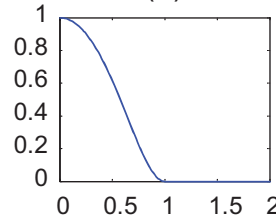

(d)

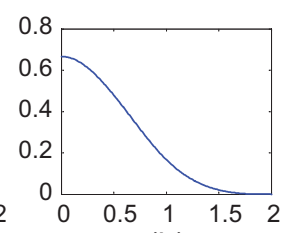

(b)

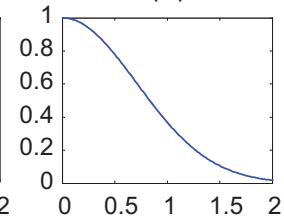

(e)

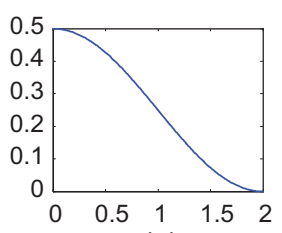

(c)

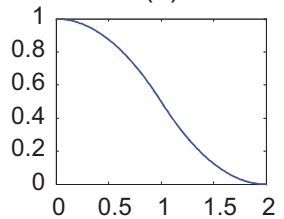

(f)
Figure 3: Kernel functions. (a)-Boxcar. (b)- Third order Spline. (c)- Hanning windowed Sinc. (d) $-f_{3},(e)-f_{1}$ and $(f)-f_{2}$ 
other kernels with such behavior. For instance, we propose the functions $f_{1}$ and $f_{2}$ shown in Figure 3.

$f_{1}(x)= \begin{cases}e^{-x^{2}} & 0 \leq x<2 \\ 0 & \text { otherwise }\end{cases}$

$f_{2}(x)= \begin{cases}\frac{2-x^{2}}{2} & 0 \leq x \leq 1 \\ \frac{(x-2)^{2}}{2} & 1 \leq x \leq 2 \\ 0 & \text { otherwise }\end{cases}$

Using these kernel functions for updating joint histogram, the mutual information function is plotted in Figure 4, as a function of translation and rotation. All methods which use sixteen pixels (i.e. GPV, HPV, $f_{1}$, and $f_{2}$ ) for interpolation have the same pattern. In particular, no local optimum is seen on the surface of the objective functions. But bilinear and PV interpolations that use only four pixels, are deeply affected by the artifacts.

Here, we use another kernel function

$f_{3}(x)=1-\sin \left(\pi x^{2} / 2\right)$

with four pixels. Although its behavior is similar to four mentioned ones [Figure 3d], its associated objective function is not smooth (like PV interpolation). Therefore, we can conclude that this is not the special kernel function which removes the artifacts in GPV and HPV methods. In fact, the number of involved pixels (which is equal to 16) is much more significant.

On the other hand, using the proposed $f_{2}$ function shown in Figure 5 , shows smoother surface in noisy images. Thus, it can be claimed that if 16 pixels are used for interpolation, there are kernel functions which removes the artifacts even better than HPV and GPV methods.

Since using 16 pixels for interpolating is a time consuming task, we are motivated to propose a new scheme which performs better than PV and bilinear interpolation methods. The distance of transformed pixel in the floating image from its four nearest pixels in the reference image is directly used in PV and bilinear interpolation methods which yields to some local optima on the surface of the mutual information function.

We try to eliminate such local optima by using the intensity of four nearest pixels besides their distances from the transformed pixel and changing the kernel function. We use $f(x)=\exp \left(\left(1-x^{2}\right) / 2\right)$ as the kernel function and multiply it by the intensity of the four nearest neighbors.

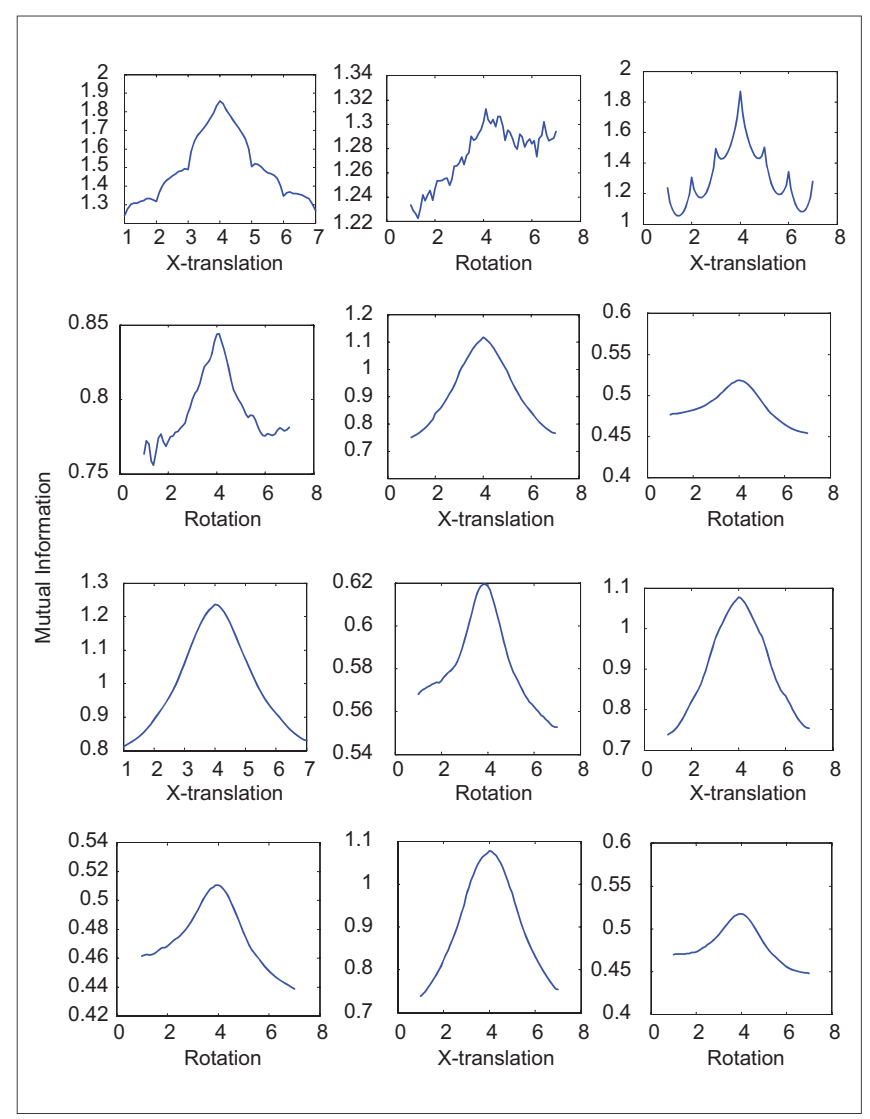

Figure 4: Typical patterns of mutual information versus rotation and translation using bilinear, PV, HPV, GPV, $\mathrm{f}_{1}$ and $\mathrm{f}_{2}$ (from first to sixth rows respectively)

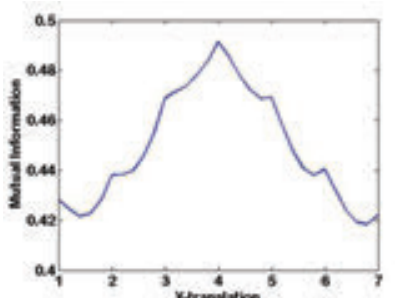

(a)

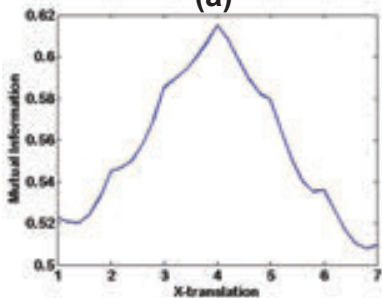

(b)

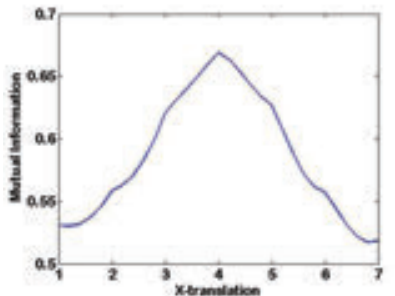

(c)

Figure 5: Patterns of mutual information versus translation using three diffrenet interpolation methods for noisy image. (a)-HPV. (b)-GPV. (c)- $f_{2}$ 
We define

$a=R\left(n_{1}\right) e^{\frac{\left(1-d_{1}\right)^{2}+\left(1-d_{2}\right)^{2}}{2}}, b=R\left(n_{2}\right) e^{\frac{\left(d_{1}\right)^{2}+\left(1-d_{2}\right)^{2}}{2}}$

$c=R\left(n_{3}\right) e^{\frac{\left(1-d_{1}\right)^{2}+\left(d_{2}\right)^{2}}{2}}, d=R\left(n_{4}\right) e^{\frac{\left(d_{1}\right)^{2}+\left(d_{2}\right)^{2}}{2}}$

$w_{1}=\frac{a}{a+b+c+d}, w_{2}=\frac{b}{a+b+c+d}$

$w_{3}=\frac{c}{a+b+c+d}, w_{4}=\frac{d}{a+b+c+d}$

Where $R\left(n_{i}\right)$ is the intensity at point $n_{i}$ and $d_{1}, d_{2}$ denote horizontal and vertical distance of the transformed point from $n_{1}$. Similar to PV algorithm, equation (3) is used to update the histogram. Note that if we use only the intensity of the pixels to calculate the joint histogram (the kernel function in equation (9) is ignored), local optima will disappear, but this type of interpolation has no strong peak in the ground truth [Figure $6 \mathrm{a}$ ].

It may seem that direct use of intensity in equation (9), makes the similarity measure sensitive to intensity variation. Certainly, the joint histogram of the images (and hence their joint probability distribution and mutual information) will be changed when the intensity of the pixels changes. This happens for all known interpolation methods. The key point is that the mutual information is robust enough (to intensity variations) so that the accuracy of registration is not affected [Figure $6 b$ and $c$ ].

Figure 7 shows mutual information function in terms of rotation and horizontal translation by $\mathrm{PV}$, bilinear and proposed interpolation methods. It can be seen that the local optima are removed with the proposed method. However, the surface of objective function is not smooth in PV and bilinear interpolation. As a result, the accuracy of registration is better with the proposed method.

\section{EXPERIMENTAL RESULTS}

For evaluating and comparing the performance of our method with PV and bilinear methods, rigid body transformation model was selected. With this assumption, only rotation and translation are permitted. Thus, for 2D images, three parameters (rotation around $\mathrm{z}$ axis and translation in $\mathrm{x}$ and $\mathrm{y}$ axis) are considered.

Note that comparison between as all of them use pixels in interpolation. We used fifth slice of CT images $(512 \times 512)$ of five patients from dataset of the project "Retrospective Image Registration Evaluation". Applying the transform function $T\left(\theta, t_{x}, t_{y}\right)$, each image was rotated at $\theta$ degrees around $\mathrm{z}$ axis, and translated in $\mathrm{x}$ and $\mathrm{y}$ directions by $t_{x}$ and $t_{y}$ pixels.

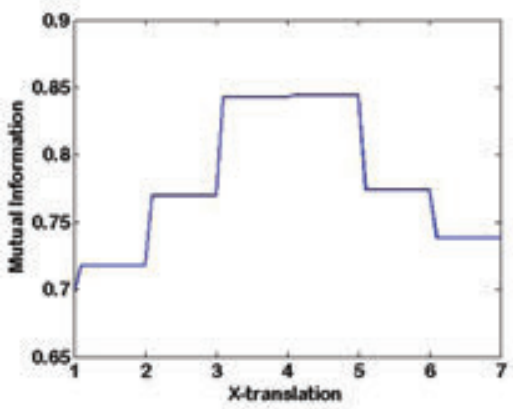

(a)

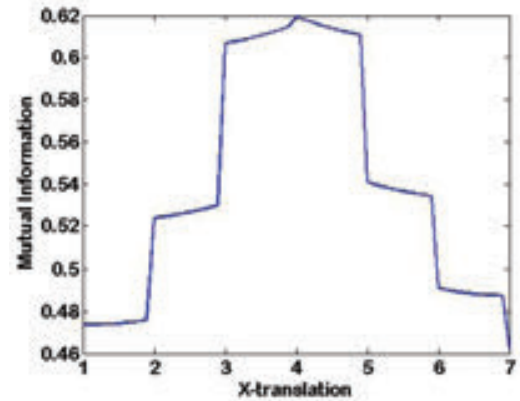

(b)

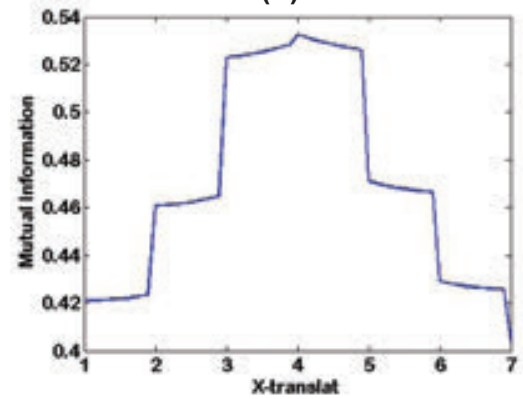

(c)

Figure 6: Pattern of mutual information in terms of translation. (a) Registration between images Figure 8 a and b by using only intensity of pixel for interpolation. (b) Registration between images Figure 8 a and b using proposed method. (c) Rgistration between image Figure 8 a and c using proposed method

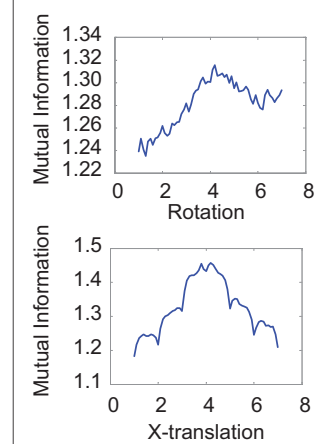

(a)
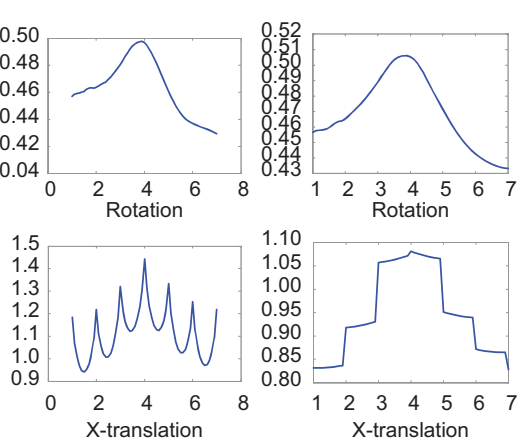

(b)

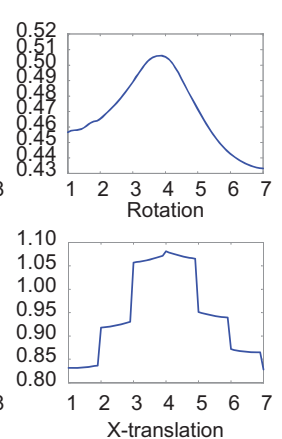

(c)
Figure 7: Patterns of mutual information versus translation and rotation using three different methods. (a)-Bilinear. (b)-PV. (c)-Proposed method

Thus, in this experiment, mutual information metric is a function in terms of three variables $\left(\theta, t_{x}, t_{y}\right)$. Zero-mean Gaussian noise with variance 0.1 was also added. The number 

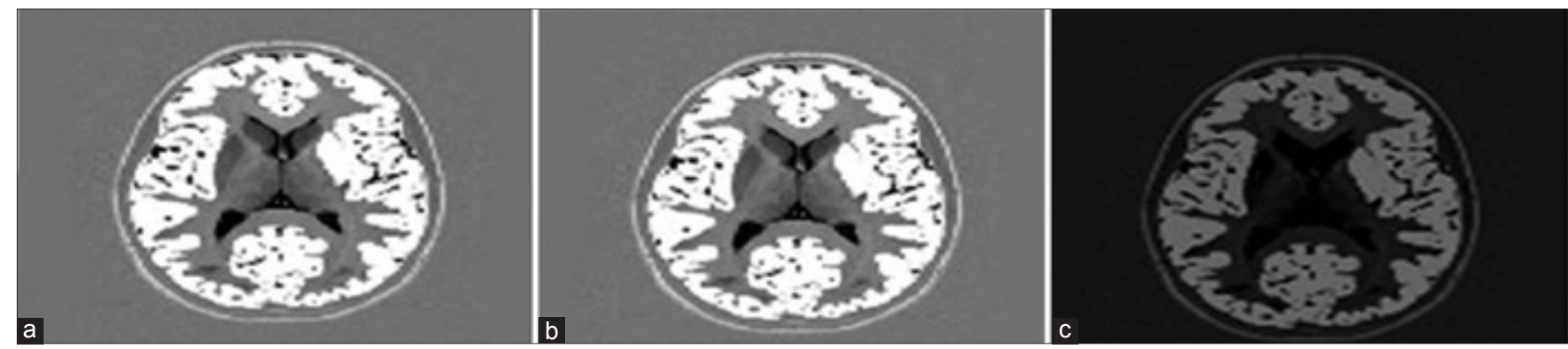

Figure 8: (a)- Original image (b)- original image translated by 4 pixels (c) original image translated by 4 pixels with decreased intensity

Table I: The result of registration using three different interpolation methods

\begin{tabular}{|c|c|c|c|c|c|c|}
\hline \multirow[t]{2}{*}{ Methods } & \multicolumn{3}{|c|}{ Parameters } & \multicolumn{3}{|c|}{ Difference } \\
\hline & $\theta$ & $t_{x}$ & $t_{y}$ & $\theta$ & $t_{x}$ & $t_{y}$ \\
\hline \multicolumn{7}{|l|}{ I } \\
\hline $\mathrm{T}$ & 3.00 & 2.50 & -3.60 & - & - & - \\
\hline $\mathrm{BI}$ & 1.34 & -0.12 & 0.03 & 1.66 & 2.62 & 3.63 \\
\hline PV & 2.94 & 2.57 & -3.43 & 0.06 & 0.07 & 0.17 \\
\hline Our method & 2.90 & 2.45 & -3.39 & 0.09 & 0.05 & 0.21 \\
\hline \multicolumn{7}{|l|}{2} \\
\hline $\mathrm{T}$ & -7.00 & 4.00 & 5.60 & - & - & - \\
\hline $\mathrm{BI}$ & 0.90 & -2.34 & 4.57 & 7.90 & 6.34 & 1.03 \\
\hline PV & -5.69 & 3.11 & 6.37 & 1.31 & 0.89 & 0.77 \\
\hline Our method & -8.17 & 3.58 & 7.02 & 1.17 & 0.042 & 1.42 \\
\hline \multicolumn{7}{|l|}{3} \\
\hline $\mathrm{T}$ & 0.00 & 2.35 & 6.85 & - & - & - \\
\hline $\mathrm{BI}$ & -0.20 & -5.60 & 5.00 & 0.20 & 7.95 & 1.85 \\
\hline PV & 0.00 & 3.00 & 7.00 & 0.00 & 0.65 & 0.15 \\
\hline Our method & 0.01 & 2.27 & 6.89 & 0.01 & 0.08 & 0.04 \\
\hline \multicolumn{7}{|l|}{4} \\
\hline $\mathrm{T}$ & 0.00 & -5.00 & 0.50 & - & - & - \\
\hline $\mathrm{BI}$ & 0.01 & -3.43 & -0.10 & 0.01 & 1.57 & 0.51 \\
\hline PV & 0.00 & -5.00 & 1.00 & 0.00 & 0.00 & 0.50 \\
\hline Our method & 0.02 & -4.98 & 0.51 & 0.02 & 0.02 & 0.01 \\
\hline \multicolumn{7}{|l|}{5} \\
\hline $\mathrm{T}$ & 0.00 & 0.00 & -7.45 & - & - & - \\
\hline $\mathrm{BI}$ & 1.21 & 0.39 & -6.41 & 1.21 & 0.39 & 1.04 \\
\hline PV & 0.00 & 0.00 & -6.00 & 0.00 & 0.00 & 1.45 \\
\hline Our method & -0.10 & 0.03 & -7.54 & 0.10 & 0.03 & 0.09 \\
\hline
\end{tabular}

$\mathrm{T}$ - Transform function; $\mathrm{BI}$ - Bilinear interpolation; PV - Partial volume

of histogram bins in all images was 128. Mutual information was used as the similarity measure and Powell-Brent method employed for optimization. Powell-Brent method is one of the popular methods. ${ }^{[16]}$ With this method, in each step of the optimization process, mutual information function is optimized in one direction of $\theta, t_{x}$ or $t_{y}$ and the resulted point in each step is used as the starting point for the next step. In the next iteration of optimization, the resulted points in the previous iteration are used. In our simulation, point $(0,0,0)$ was considered as the starting point and variation of parameters $\left(\theta, t_{x}, t_{y}\right)$ was limited to the interval $(-10,10)$. The result of this simulation is shown in Table 1 .

It can be seen that both of the proposed methods and PV interpolation give an estimation of transformation function ' $T$ ' better than that of the bilinear interpolation. But when
Table 2: The result of registration using PV and proposed interpolation methods

\begin{tabular}{|c|c|c|c|c|}
\hline \multirow[t]{2}{*}{ Methods } & \multicolumn{2}{|c|}{ Parameters } & \multicolumn{2}{|c|}{ Difference } \\
\hline & $t_{x}$ & $t_{y}$ & $t_{x}$ & $t_{y}$ \\
\hline \multicolumn{5}{|l|}{ I } \\
\hline $\mathrm{T}$ & 3.00 & -3.00 & - & - \\
\hline PV & 1.00 & -2.00 & 2.00 & 1.00 \\
\hline Our method & 3.03 & -3.00 & 0.03 & 0.00 \\
\hline \multicolumn{5}{|l|}{2} \\
\hline $\mathrm{T}$ & 7.00 & 5.00 & - & - \\
\hline PV & 5.00 & 5.00 & 2.00 & 0.00 \\
\hline Our method & 6.85 & 5.00 & 0.25 & 0.00 \\
\hline \multicolumn{5}{|l|}{3} \\
\hline $\mathrm{T}$ & -6.00 & 9.00 & - & - \\
\hline PV & -3.00 & 5.00 & 3.00 & 4.00 \\
\hline Our method & -6.00 & 9.25 & 0.00 & 0.25 \\
\hline \multicolumn{5}{|l|}{4} \\
\hline $\mathrm{T}$ & 1.00 & -1.00 & - & - \\
\hline PV & 1.00 & -1.00 & 0.00 & 0.00 \\
\hline Our method & 1.02 & -1.00 & 0.02 & 0.00 \\
\hline \multicolumn{5}{|l|}{5} \\
\hline $\mathrm{T}$ & 8.00 & 8.00 & - & - \\
\hline PV & 5.00 & 6.00 & 3.00 & 2.00 \\
\hline Our method & 8.00 & 7.00 & 0.00 & 1.00 \\
\hline
\end{tabular}

the images are not rotated (or $\theta$ is zero), the proposed method has high accuracy and fewer errors. This is because the PV interpolation artifacts are the direct results of using the simple boxcar kernel function and when $\theta=0$ local optima will appear for integer pixel shifts. Such artifacts are not so evident when $\theta$ is not zero. ${ }^{[17]}$ As seen in Figure 8, local optima are in integer translations and the resulted estimations for patients 3,4 , and 5 ( $\theta$ is zero for these patients) which are given in Table 1 , confirms this.

To clarify the superiority of the proposed method, we set $\theta=0$ in another experiment. Original images were added by zero-mean Gaussian noise with variance 0.01 . Optimization was performed to drive optimal value of $t_{x}$ and $t_{y}$. The results of this simulation are given in Table 2.

It can be seen that with PV interpolation method, the optimization algorithm converges to one of the local optima (in integer translation), which is shown in Figure 8. However, due to removing artifacts, the proposed method shows higher accuracy. 


\section{CONCLUSION}

In this paper, the intensity-based image registration methods which use mutual information as similarity measure and the effect of artifacts resulted by PV and bilinear interpolation methods were studied. Also, we considered GPV and HPV interpolation methods that use 16 pixels for interpolating. By introducing two new functions, $f_{1}$ and $f_{2}$ as kernel functions, we showed that the acceptable performance of GPV and HPV is because of using 16 pixels not using special kernel functions. Since methods which use 16 pixels for interpolation are time consuming, we proposed a new interpolation method that use four pixels to interpolate images and removes local optima (artifacts) from the objective function.

\section{REFERENCES}

1. J.B.A. Maintz and M.A. Viergever. A Survey of Medical Image Registration. Medical Image Analysis 2, pp. 1-36, 1998.

2. C.R. Maurer Jr. and J.M. Fitzpatrick. A Review of Medical Image Registration, in Interactive Image-Guided Neurosurgery, R.J. Maciunas, Ed. pp. 17-44. American Associate of Neurological Surgeons, Park Ridge ILUSA, 1993.

3. D. Loeckx, P. Slagmolen, F. Maes, D. Vandermeulen, and P. Suetens. Non rigid Image Registration Using Conditional Mutual Information. IEEE transactions on medical imaging, Vol. 29, NO. 1, JANUARY 2010.

4. B. Zitova, J. Flusser. Image registration methods: A survey. Image and Vision Computing 21, pp. 977-1000, 2003.

5. A. Gholipour, N. Kehtarnavaz, R Briggs, M Devous, K Gopinath. Brain Functional Localization: A Survey of Image Registration Techniques. IEEE transaction in medical imaging 26, pp. 427-51, 2007.

6. X. Lu, Su. Zhang, H. Su and Y. Chen. Mutual information-based multimodal image registration using a novel joint histogram estimation. Computerized Medical Imaging and Graphics 32, pp. 202-209, 2008.
7. N. Dowsen, T. Kadir, and R. Bowden. Estimating the Joint Statistics of Images Using Nonparametric Windows with Application to Registration Using Mutual Information. IEEE transaction on pattern analysis and machine intelligence 30, pp. 1841-1857, 2008.

8. R. Shams, P. Sadeghi, R.I. Hartley. A Survey of Medical Image Registration on Multi-core and the GPU. IEEE signal processing 11 , pp. 50-60, 2010.

9. R. Brunelli. Template Matching Techniques in Computer Vision Theory and Practice. United States: J. Wiley and Sons; 2009.

10. N. Dowson and R. Bowden. Simultaneous modeling and tracking (smart) of feature sets. Computer Vision and Pattern Recognition, Vol.2, pp. 99-105, 2005.

11. F. Mase, A. Collignon, D. Vandermeoulen, G. Marchal and D. Suet. Multimodality image registration by maximization of mutual information. IEEE Transaction on Medical Imaging 16, pp. 187-198, 1997.

12. P.W. Pluim, J.B. Maintz, and A. Viergever. Interpolation Artifacts in Mutual Information-Based Image Registration. Computer Vision and Image Understanding 77, pp. 211-232, 2000.

13. H. Chen, P.K. Varshney. Mutual information-based CT-MR brain image registration using generalized partial volume joint histogram estimation. IEEE Transaction in Medical Imaging 22, pp. 111-119, 2003.

14. P. Viola and W.M. Wells. Alignment by maximization of mutual information, in International Conference on Computer Vision. (E. Grimson, S. Shafer, A. Blake, and K. Sugihara, Eds.), IEEE Computer Society Press: Los Alamitos, CA; pp. 16-23, 1995.

15. A. Papoulis and S.U. Pillai, Probability, Random Variables, and Stochastic Processes. third ed., New York: McGraw-Hill; pp. 124-148, 1991.

16. W. Press, S. Teukolsky, W. Vetterling, and B. Flannery. Numerical Recipes in C, second. Cambridge: Cambridge University Press; 408-420, 2002.

17. J. Xiuquan, H. Pan, and Z.P. Liang. Further Analysis of Interpolation Effects in Mutual Information-Based Image Registration. IEEE transaction on medical imaging 22, pp. 1131-40, 2003.

How to cite this article: ***

Source of Support: Nil, Conflict of Interest: None declared

\section{BIOGRAPHIES}

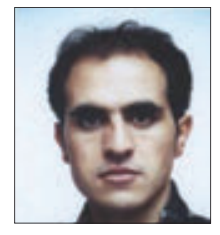

Hossein Soleimani received the B.Sc. and M.Sc. degree from Shahid Rajayi Teacher training University, Tehran, Iran, in 2008 and Isfahan University of Technology in 2011 respectively, both in electrical Engineering. During his four years at Shahid Rajayi Teacher Training University his research was in neural network systems and image processing. His interests in Isfahan University of Technology include image and signal processing and medical imaging.

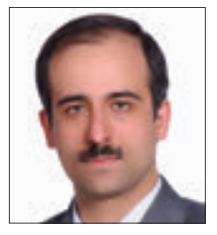

Mohammadali Khosravifard received the B.Sc., M.Sc., and Ph.D. degrees in electrical engineering from Shiraz University, Sharif University of Technology, and Isfahan University of Technology, Iran, in 1996, 1998, and 2004, respectively. Since 2004, he has been an Assistant Professor with the Department of Electrical and Computer Engineering, Isfahan University of Technology. His main research interests include information theory and related topics in image processing. 